\title{
Didáctica para la Enseñanza del Concepto de Valencia Química
}

\author{
Candelaria Tejada ${ }^{(1) \star}$, Diofanor Acevedo ${ }^{(2)}$, Aníbal Mendoza ${ }^{(3)}$ \\ (1) Universidad de Cartagena, Facultad de Ingeniería, Programa de Ingeniería Química, Avenida el \\ Consulado, Calle 30 No. 48-152. Cartagena, Bolívar-Colombia (e-mail: candelariatejada@yahoo.com) \\ (2) Universidad de Cartagena, Facultad de Ingeniería, Programa de Ingeniería de Alimentos, Avenida el \\ Consulado, Calle 30 No. 48-152. Cartagena, Bolívar-Colombia \\ (3) Universidad del Norte, Departamento de Física, Km 5, Vía a Puerto Colombia. Barranquilla, Atlántico- \\ Colombia.
}

* Autor a quien debe ser dirigida la correspondencia

Recibido Dic. 22, 2014; Aceptado Feb. 16, 2015; Versión final Mar. 28, 2015, Publicado Oct. 2015

\begin{abstract}
Resumen
Se propone una herramienta didáctica para la enseñanza del concepto de valencia química. Con este fin, se realizó un diagnóstico inicial entre una muestra de docentes sobre la forma como enseñan y evalúan el concepto de valencia en química, así como las características del concepto que tienen en cuenta para su enseñanza. De igual forma se indagó sobre las estrategias pedagógicas y metodológicas utilizadas en el aula, que favorezcan en el estudiante el aprendizaje de este concepto. De estos resultados emergieron algunas de las características que permitieron la construcción del concepto de valencia. Esto se complementó con el ejercicio que hicieron los estudiantes al realizar las cuatro operaciones intelectuales: el supraordenado, el infraordenado, la isordinada, y el excluyente. Todas las características identificadas y seleccionadas para este caso específico, se organizaron con el fin de proponer un mentefacto conceptual (representación gráfica) como herramienta para enseñar el concepto de valencia.
\end{abstract}

Palabras clave: didáctica, valencia química, valencia secundaria, mentefacto, aprendizaje significativo

\section{Educational Resources for Teaching the Concept of Chemical Valence}

\begin{abstract}
The present study was aimed to propose an educational tool for teaching the concept of chemical valence. For this, an initial diagnosis among a sample of teachers on how they teach and evaluate the concept of valence chemistry was performed, and the concept features taken into account in their teaching. Likewise questions were asked about the pedagogical and methodological strategies used in the classroom, which encourage student learning concept. From these results some of the features that enabled the construction ho of the concept of valence emerged. This was complemented with exercises done by the students who perform the four intellectual operations: super-organized, sub-organized, organized and exclusive. All features identified and selected for this specific case, were organized to propose a conceptual graphical representation (mentefacto) as a tool to teach the concept of valence.
\end{abstract}




\section{INTRODUCCIÓN}

En la mayoría de los textos de química general, el concepto de valencia química es manejado de manera tradicional, en forma incompleta y estática; lo que contribuye a la desarticulación curricular del concepto de valencia; en la mayoría de casos los profesores no son conscientes de las limitaciones que se generan en los estudiantes en el proceso de comprensión de la ciencia química, como consecuencia de abordar únicamente el concepto de valencia primaria en los cursos de química básica; lo que según lo propuesto por (Gallego et al., 2004), con ello se limita la construcción de estructuras cognitivas sólidas en química, imposibilitando el aprendizaje significativo. Es decir con este concepto solo se posibilita la explicación y comprensión de algunas moléculas (la minoría de ellas) en la que únicamente es posible la interacción de los electrones del último nivel de energía para su formación, y en lo cual participan sólo elementos de los grupos representativos de la tabla periódica, sin que se permita explicar todos los compuestos que pueden existir.

Según Dos Santos-Fernandes y Fernandes-Campos (2014) además de estas dificultades, también se revela la incapacidad de relacionar este tipo de concepto con los tres niveles de conocimiento químico, representacionai, macroscópico y microscópico. Según este mismo autor, estas barreras, una vez identificadas por el profesor, abren posibilidades para el desarrollo de estrategias de enseñanza que las superen. En este sentido, este estudio propone el mentefacto como una didáctica de enseñanza en clases que permita integrar el concepto de valencia secundaria al concepto tradicionalmente enseñado. Algunas investigaciones ya se han realizado vinculado instrumentos didácticos para la enseñanza de conceptos de la ciencias básicas (Carneiro y Dal Farra, 2011; Latasaet al., 2012; Lorenzo et al., 2011) y, particularmente, en la enseñanza de la química (Green et al., 2012; Simõeset al., 2013; Vega et al., 2014; Green y Parkin, 2014).

Un mentefacto es un diagrama jerárquico cognitivo que organiza y preserva el conocimiento, en él se plasman las ideas fundamentales y se desechan las secundarias (Ibañez, 2006). Según De Zubiría (2006), los mentefactos conceptuales poseen cuatro cuestiones (supraordinada, infraordinadas, excluyentes y características, que son las isoordinadas), que resultan capitales para caracterizar un concepto en un modelo sencillo y estético, en donde la clase superior aparece arriba, las clases excluidas a la derecha; las cualidades esenciales a la izquierda y las versiones o variantes del concepto aparecen abajo. Es pertinente traer a colación los instrumentos fundamentales del saber conocer, los cuales se muestran en la Tabla 1 de Tobón(2005),el cual se fundamenta en la teoría planteada por De Zubiría (2006).

Lo anterior invita a reflexionar que en las universidades colombianas tienen que empezar urgentemente a liderar procesos de transformación en sus procesos formativos, ya que el nuevo mundo laboral no requiere de tanta mano de obra, si no, de cerebros pensantes, específicamente en nuestro caso, personas verdaderamente inteligentes, lo que De Zubiría (2006) llama analistas simbólicos.

Tabla 1: Instrumentos fundamentales del saber conocer

\begin{tabular}{|l|l|l|l|}
\hline $\begin{array}{c}\text { Instrumento } \\
\text { cognitivo }\end{array}$ & \multicolumn{1}{|c|}{ Definición } & \multicolumn{1}{|c|}{ Ejemplos } & Procesos implicados \\
\hline Nociones & $\begin{array}{l}\text { Representaciones de la realidad, las } \\
\text { cuales se estructuran por palabras e } \\
\text { imágenes interrelacionadas entre si }\end{array}$ & $\begin{array}{l}\text { Grande-pequeño } \\
\text { Fuera-dentro } \\
\text { Blanco-negro }\end{array}$ & $\begin{array}{l}\text { Proyectar } \\
\text { Introyectar } \\
\text { Comprender } \\
\text { Nominar }\end{array}$ \\
\hline Proposiciones & $\begin{array}{l}\text { Son aseveraciones acerca de clases } \\
\text { generales de la realidad con base en } \\
\text { un sujeto y un predicado }\end{array}$ & $\begin{array}{l}\text { "las competencias se componen } \\
\text { de tres saberes esenciales: } \\
\text { saber ser. Saber conocer y } \\
\text { saber hacer" }\end{array}$ & $\begin{array}{l}\text { Proposicionalizar } \\
\text { Ejemplificar } \\
\text { Codificar } \\
\text { Decodificar }\end{array}$ \\
\hline Conceptos & $\begin{array}{l}\text { Representan un conjunto organizado } \\
\text { de abstracciones }\end{array}$ & $\begin{array}{l}\text { Capacidad } \\
\text { Pensamiento } \\
\text { Aprendizaje } \\
\text { Competencia }\end{array}$ & $\begin{array}{l}\text { Supraordinar } \\
\text { Infraordinar } \\
\text { Isoordinar } \\
\text { Excluir }\end{array}$ \\
\hline Categorías & $\begin{array}{l}\text { Son tejidos de conceptos que se } \\
\text { construyen mediante procesos de } \\
\text { argumentación y derivación }\end{array}$ & $\begin{array}{l}\text { Sistema educativo } \\
\text { Sistema enseñanza-aprendizaje }\end{array}$ & $\begin{array}{l}\text { Argumentar } \\
\text { Derivar } \\
\text { Definir } \\
\text { Subargumentar }\end{array}$ \\
\hline
\end{tabular}

Por lo tanto, se hace necesario reinventar procesos cognitivos y formas de agilizar el conocimiento, de manera tal que el estudiante pueda generar procesos más activos de aprendizaje. Es así como en el presente trabajo nos centramos en el saber conocer, específicamente, el concepto de valencia, esto implica que de alguna manera nos tenemos que detener a pensar, si valencia es un concepto, pero somos conscientes que para poder realizar el aprendizaje significativo de un concepto el estudiante debe estar en capacidad de identificar 
cuatro componentes, o de realizar cuatro operaciones intelectuales: el supraordenado, el infraordenado, la isordinada y lo que lo diferencia de otros conceptos, es decir el componente excluyente.

El presente trabajo de investigación se enfocó en identificar y proponer una didáctica de enseñanza en clases que permita integrar el concepto de valencia secundaria al concepto tradicionalmente enseñado, puesto que actualmente, este concepto se deja para cursos de química avanzada, conocimiento al que muchos estudiantes nunca accesan, y con el cual es posible explicar la formación de todos los compuestos inorgánicos incluyendo los compuestos de coordinación donde participan químicamente los elementos de los grupos de transición, cuya existencia no explica la valencia primaria.

\section{MATERIALES Y MÉTODOS}

La presente investigación es de tipo descriptiva, en donde la información recolectada durante la implementación de la didáctica por parte de docentes para enseñar el concepto de valencia química fue analizada bajo la estructura del modelo de Harry F. Wolcott. Wolcott. (1994), citado en Lucca N., Berrios R., (2003); que consta de tres partes: descripción, análisis e interpretación, articulándolas de manera simultánea al presentar los resultados.Según Lucca y Berrios (2009) la descripción contiene diez modos distintos de organización de la información. En el presente trabajo se realizó desde la denominada "focalización progresiva", en el cual se tiene en cuenta la información obtenida desde la actividad desarrollada en la primera sesión de clases (Base orientadora de la Acción), hasta la cuarta sesión (proposición de la didáctica).

\section{Participantes}

Cinco docentes voluntarios del área de química de programas de ingeniería a nivel Universitario, para realizar un diagnóstico inicial, sobre la forma como se está enseñando en la actualidad el concepto de valencia. Estos docentes fueron seleccionados de las diferentes carreras donde se imparten asignaturas del área de química en la Universidad de Cartagena (Colombia). Además, tres investigadores; representados en un observador participante y dos observadores no participantes, que triangularon información recopilada de un grupo de 30 estudiantes de química inorgánica de la facultad de ingeniería de la misma universidad.

\section{Procedimiento}

Se realizó un diagnóstico inicial entre los docentes sobre la forma como enseñan y evalúan el concepto de valencia en química, así como las características del concepto que tienen en cuenta para su enseñanza. De igual forma se indagó sobre las estrategias pedagógicas o metodológicas utilizadas en el aula, que favorezcan en el estudiante el aprendizaje de este concepto. Esto mediante la aplicación de entrevistas semiestructuradas, con las cuales también se identificó las concepciones que tienen los docentes acerca del concepto de valencia. De estas concepciones se realizó un análisis y una interpretación de resultados de donde emergieron algunas de las características que permitieron la construcción del concepto de valencia complementado con el ejercicio que hicieron los estudiantes al realizar las cuatro operaciones intelectuales: el supraordenado, el infraordenado, la isordinada y lo que lo diferencia de otros conceptos, es decir el componente excluyente. A estos también se les aplicó un test de conceptos previos en la clase de formación de moléculas sencillas y complejas del curso de inorgánica, todo esto apoyado en la revisión de la evolución histórica del concepto por parte del docente y los estudiantes, así como la capacitación sobre la construcción de mentefactos y su utilidad. Todas las características identificadas y seleccionadas de las dos fuentes se plasmaron en el mentefacto propuesto para este caso específico, con el fin de proponer una herramienta para enseñar un concepto.

\section{RESULTADOS Y ANÁLISIS}

Luego de identificar las concepciones que tienen los docentes acerca del concepto de valencia, es importante resaltar la opinión de los entrevistados.El primer docente afirma: "Para mí el concepto de valencia ha caído en desuso, prácticamente no lo uso por que cada día se vuelve más rígido y según la descripción antigua de valencia, que era la capacidad que tenía un átomo para enlazarse, la capacidad de enlace de un átomo sería un concepto muy rígido en la medida en que han salido nuevos conceptos. Hoy hay un concepto de combinación, concepto de estado de oxidación, que se ha ampliado más y que le dan una mayor flexibilidad a esta capacidad de enlace fundamental"; lo cual permite inferir que este profesor en su concepción muy particular del concepto de valencia, tiene claridad de que la forma actual como se viene enseñando, no es la adecuada, pues se sigue manejando la definición antigua de capacidad de enlace. Lo que probablemente está limitando al estudiante en su posibilidad de concebir, entender y poder explicar la creación de nuevos enlaces, pues se le hace difícil pensar en todas las posibilidades que ofrece la naturaleza y que a través de síntesis se puedan crear nuevos compuestos. Cuando el docente afirma que, "no estoy de acuerdo en hacer el concepto rígido, por ejemplo, la coordinación es un concepto que explica mucho mejor, le da mucha 
másamplitud a estos enlaces", se puede percibir que su concepción respecto al concepto de valencia es amplio y completo, pues cuando incluye el concepto de coordinación de un compuesto indica que a lo mejor tiene claridad sobre los conceptos de valencia primaria y valencia secundaria, que tiene en cuenta el desarrollo histórico del concepto, lo cual vuelve el concepto más flexible.

Del análisis de la entrevista suministrada por el Docente \#1, se pudo construir una categoría que agrupa a los docentes que no están de acuerdo con la enseñanza del concepto tradicional de valencia química (Categoría 1). De lo anterior,se extractaronalgunas concepciones a manera deproposiciones: 1) En la enseñanza de la química no es útil hacer énfasis en el antiguo concepto de valencia, 2) El concepto rígido-antiguo de valencia no permite la comprensión de otros conceptos, 3) Para mejorar la enseñanza del concepto de valencia hay que asociarla a otros conceptos, como numero de oxidación y numero de coordinación, 4) Hay conceptos asociados al concepto de valencia que le permiten su flexibilidad, 5) Ante las posibles probabilidades de enlace de un átomo, el concepto de valencia como lo muestran los textos es muy rígido y 5) La existencia de muchas moléculas son inexplicables con el concepto tradicional de valencia.

También se consideró pertinente citar algunas afirmaciones que hace el segundo docente, como por ejemplo "valencia simplemente, es el número de uniones que puede hacer un átomo con otros átomos pero, digamos que uno tiene que contextualizarlo dentro de todo lo que acabo de decir." Este docente a diferencia del se enfocaen el concepto tradicional de valencia que tanto critica el Docente \#2, es decir no contempla los aspectos que se consideran relevantes del docente anterior, no complementó ni tampoco realizó ninguna crítica al concepto actual, sin embargo elDocente \#2 considera muy importante la inclusión del desarrollo histórico y epistemológico en la enseñanza del concepto de valencia, lo que se dedujo de su afirmación: "Para llegar al concepto uno tiene primero, que partir de una especie de evolución histórica y decir cuáles son las diferentes teorías de la estructura de la materia, para esto tendríamos que hablar de los primeros que hablaron sobre ello como los Griegos, Dalton, las teorías de Thomson y luego Bohr y el actual modelo de la materia, que es el modelo de la química cuántica". Esto toma relevancia en tanto que los investigadores en didáctica de las ciencias han concluido que la inclusión de la historia de las ciencias en su enseñanza es de una alta importancia, ya que esta puede aportar a solucionar problemas tales como la visión distorsionada y los reduccionismos a los que la ciencia ha sido expuesta. López E, \& Ospina J., (2011), y teniendo en cuanta que con la inclusión de la historia de las ciencias en su enseñanza se puede establecer un hilo conductor que da cuenta del progreso científico y, a su vez, identificar los conceptos estructurantes que permiten la introducción de otros conocimientos para, de esta forma, poder proponer soluciones ante los obstáculos epistemológicos. Tejada C et al., (2013).

Del análisis de la entrevista suministrada por el Docente\#2, se identificó una segunda categoría, representada por los profesores que comparten la enseñanza del concepto tradicional de valencia química, (rígido y acabado), teniendo en cuenta su evolución histórica, categoría de la que se sintetizan unas concepciones a manera deproposiciones: 1) El concepto de valencia se limita soloenel estudio de los electrones de las últimas capas energéticas de un átomo, 2) Valencia es simplemente el número de uniones que puede hacer un átomo con otros átomos y 3) Para comprender el concepto de valencia hay que contextualizarlo en la evolución histórica explicativa de la estructura de la materia y de la organización de la tabla periódica.

Dada las grandes diferencias entre las respuestas de los docentes entrevistados, también es pertinente citar algunas afirmaciones del Docente \#3, quien da muestra de manejar el concepto de manera rígida e igual que el Docente \#2; cuando afirma que: "El concepto de valencia es la capacidad que tiene un elemento de combinarse conotro elemento". A diferencia del Docente \#2, el \#3 no menciona el desarrollo histórico de concepto, lo que de igual manera obstaculiza la comprensión de la química, en lo referente a la existencia de muchas moléculas inorgánicas, que con este concepto, no serían posibles deexplicar y ni siquiera se le da la posibilidad al estudiante de pensar en que puedan existir dichas moléculas, pues el concepto se presenta de una manera plana y sin otras posibilidades de exploración.

En este mismo sentido losDocente \#4 y \#5, también conciben el concepto de valencia de manera rígida.Ambos afirman que: "el concepto de valencia es la capacidad que tiene un elemento de combinarse conotro elemento"; pero a diferencia de los dos anteriores, estos, lo relacionan con enlace químico y tabla periódica, sin mencionarel desarrollo histórico. Del análisis de las entrevistas suministradas por los Docente \#4 y \#5 se pudo conformar la tercera categoría de quienes comparten la enseñanza del concepto tradicional de valencia química (rígido y acabado), relacionándolo conel concepto de tabla periódica y enlace químico, evidenciado las siguientes proposiciones: 1) El concepto de valencia es la capacidad que tiene un átomo o un elemento de combinarse con otros elementos de acuerdo a su carga positiva o carga negativa, 2) $\mathrm{EI}$ concepto de valencia tiene que ver con el concepto de tabla periódica, con la organización de metales y no metales, 3) La tabla periódica está organizada de tal maneraque se puede saber que tantas posibilidades tiene un elemento para combinarse con otro, 4) Los estados de oxidación dan información sobre la carga positiva o negativa de un átomo, 5) El concepto de valencia está relacionado con la capacidad que tiene un 
átomo para formar enlaces, 6) La valencia de un átomo está relacionado con la ganancia, pérdida o comparticiónde electrones, y es una propiedad periódica, 7) La valencia es la que indica el tipo de enlace que puede formar un átomo, 8) Del concepto de valencia se derivan los conceptos de enlace y de reacción química y El concepto de valencia es importante pero los profesores solo lo manejan a manera de definición, se trae a colación cuando se necesita para explicar otro concepto como enlace.

\section{Similitudes encontradas}

Haciendo un análisis comparativo de las cinco entrevistas se encuentran aspectos en común tales como: 1) Los tres docentes asocian el concepto de valencia a otros conceptos relacionados con la estructura de la materia, 2) Los Docentes\#1 y \#2 consideran que el concepto de valencia se está enseñando de manera aislada, lo que le da cierta rigidez enel engranaje de la comprensión de la química y 3) Los Docentes \#2, \#3, \#4 y \#5 enseñan en concepto de valencia de manera tradicional, como lo presentan los textos, es decir rígidoy acabado, concibiéndolo únicamente como la capacidad de enlace que tiene un átomo.

\section{Diferencias encontradas}

El Docente \#1 muestra una visión amplia y actualizada de lo que implica la capacidad de enlace de los átomos, al relacionarlos con conceptos como: números de oxidación y de coordinación. Lo anterior permite al estudiante considerar las diferentes posibilidades de enlace que pueden tener los átomos acorde con lo que se presenta en la naturaleza; mientras que los Docente \#2 y \#3, enseñan el conceptode valencia fundamentado en el estudio de los electrones presentes en el último nivel de cada átomo.La diferencia entre elDocente \#2 y los Docente \#3, \#4 y \#5 está en que el Docente \#2 enseña el concepto de valencia en un contexto histórico, lo que le permite al estudiante valorar los aportes que han suministrado diferentes investigadores a través de la historia en la construcción del concepto, y por su parte el resto de los docentes lo enseñan de una forma muy limitada y tradicional.

La enseñanza del concepto por los cinco docentes se hace de manera diferente: el Docente \#1 no lo enseña específicamente porque lo considera en desuso, le encuentra más aplicabilidad al concepto de coordinación; el Docente \#2 utiliza como estrategia didáctica la contextualización histórica del concepto tradicional, el Docente \#3 se apoya en la regla del octeto; y los Docente \#4 y \#5 también enseñan el concepto tradicional pero de manera periférica, pues enseñan otros conceptos como enlace químico y/o tabla periódica. Es importante resaltar que el Docente \#4 considera importante la parte histórica y epistemológica del concepto, pero no la incluye en sus técnicas de enseñanza por falta de tiempo, como se puede observar en la cita siguiente: "yo siempre he dicho que son las reglas del juego de la química (refiriéndose a la inclusión de la historia), la verdad es que por el tiempo que uno tiene no profundiza mucho en la historia de la química, pero la base de la tabla periódica está en la historia de la química". Referente a lo anterior, en Colombia los aspectos de tipo histórico se encuentran ausentes en la mayoría de los libros de texto y que, cuando aparecen son tratados en forma superficial, sin atribuirles un papel relevante, igual a como ocurre en otros países; lo cual, de acuerdo a Gallego (2004), "sumado al papel del desempeño del profesor como instrumento transmisor acrítico de los contenidos curriculares, podría ser una de las causas del desinterés de los alumnos por el estudio de las ciencias".

También es importante resaltar que el Docente \#5 considera el concepto de valencia muy importante, sin embargo en la asignatura de química general no le asigna un espacio exclusivo para enseñarlo, como se puede ver en la siguiente cita: "en realidad es un concepto importante porque de él se derivan los enlaces y por lo tanto las reacciones químicas, sin embargo es un concepto que a veces los profesores pasamos por alto simplemente damos una definición luego cuando estamos en enlace nuevamente nos remitimos a el como vimos el concepto de valencia, pero en realidad se explica con muy pocos detalles".

Lo anterior denota una gran disparidad en materia curricular, pues, unos docentes incluyen la enseñanza del concepto como parte del contenido, mientras que otros no, estos últimos nolo enseñan, sino que lo traen a colación de manera periférica como apoyo en la enseñanza de otros conceptos como tabla periódica o enlace químico; muy a pesar de lo conscientes que son de la importancia de la inclusión del concepto de valencia dentro de la enseñanza de la química, como lo expresó el Docentes \#5.

\section{El Mentefacto como didáctica propuesta para la enseñanza del concepto de valencia}

Después de analizar las características propuestas por los estudiantes en el test de conceptos previos y de analizar los mentefactos construidos por los estudiantes; los autores propusimos un mentefacto del concepto de Valencia, como una alternativa de didáctica para la enseñanza del concepto, que incluye elementos identificados en el análisis del desarrollo histórico y epistemológico del concepto. Todo esto se suministró en la cuarta clase a los estudiantes el cual es un producto propuesta para este estudio de caso específico, la 
propuesta que se hace, se muestra en la Figura 1. Y está fundamentada desde el punto de vista disciplinar y con el objeto de proponer una herramienta didáctica que emerge de la teoría de las acciones mentales de Galperin, (1983), y las reglas mentefactuales expuestas por De Zubiría (2006).

En el ejercicio que hicieron los estudiantes al realizar las cuatro operaciones intelectuales: se identificó como el supraordenado (atomo enlazante), el infraordenado (valencia primaria y valencia secundaria), la isordinada identificada como las características del concepto (Pérdida o ganancia o compartición de electrones, número de oxidación, ley del octeto, intervienen los electrones del último nivel de energía, capacidad de combinación de un átomo, numero de coordinación, número de electrones que participan en un enlace, promoción de electrones de niveles internos de energía, interacción de ligandos con un centro metálico, numero de enlaces que puede formar un átomo para formar una molécula) y por último se identificó el componente excluyente, es decir y lo que lo diferencia de otros conceptos, como (numero atómico, numeró másico, propiedades periódicas, estructura atómica, peso atómico, potencial de ionización, electronegatividad, propiedades físicas, radio atómico, y afinidad electrónica).

Desde el punto de vista pedagógico a los estudiantes se les debe presentar en la primera clase un test de conceptos previos, para identificar la concepción que tienen del concepto, así como sus errores conceptuales, en la segunda clase capacitarlos sobre el mentefacto, sus componentes su utilidad y forma de construirlo, seguido de una presentación del desarrollo histórico del concepto a enseñar, en este caso el concepto de valencia química, en la tercera clase se les presenta la clase magistral sobre formación de moléculas inorgánicas sencillas y complejas y toda la parte conceptual de soporte y en la cuarta y última clase se les realiza la actividad de la construcción del concepto de valencia.

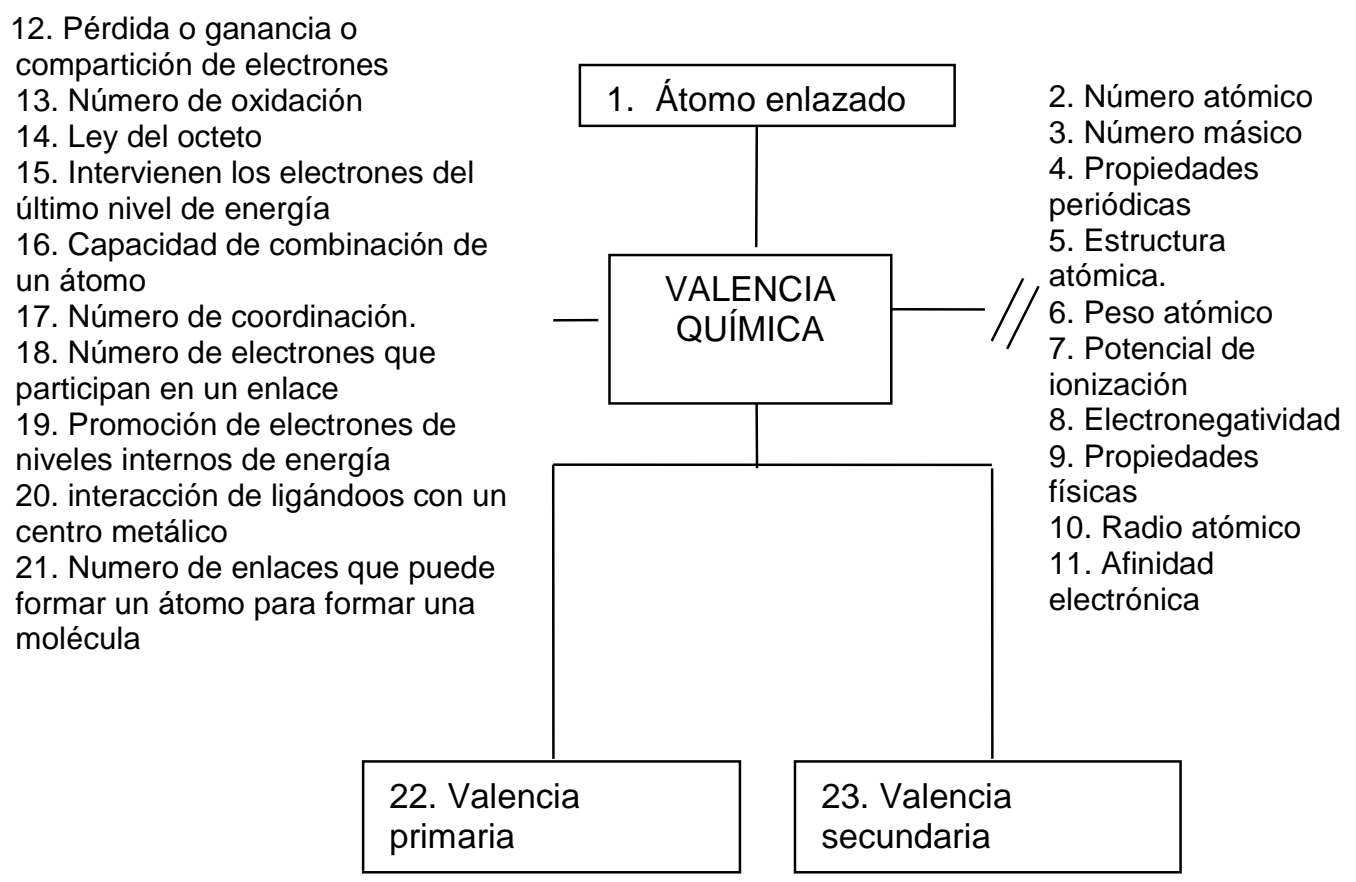

Fig. 1: Mentefacto del concepto de valencia propuesto por los investigadores

\section{CONCLUSIONES}

Respecto a las entrevistas realizadas se identificaron tres perfiles de docentes con respecto a la enseñanza del concepto de valencia. El Docente \#1 muestra un perfil con conceptos actualizados y que se preocupa por que el estudiante pueda explicar la existencias de muchas moléculas, que enseñadas con el concepto tradicional, no tendrían explicación, lo que limita en gran medida la capacidad de entendimiento de estas moléculas para el estudiante. El Docente \#2 se preocupa por contextualizar históricamente la enseñanza del concepto de valencia, aunque maneja la enseñanza del concepto tradicional y lo entiende de manera simple, como la capacidad que tienen los átomos para formar enlaces. El último perfil lo tienen los Docente \#3, \#4 y \#5; y corresponde al docente tradicional que enseña el concepto de manera rígida apoyado en la regla del octeto y no da muestras de preocuparse por que el estudiante entienda la existencia de otra moléculas, quizás porque no es consciente de ello; también lo relaciona con otros conceptos como enlace químico o tabla periódica. 
Los investigadores pudimos identificar cuatro causas por las cuales los docentes enseñan cosas diferentes y con profundidad diferente, relacionadas con los perfiles identificados anteriormente:

El Docente \#1 aborda la enseñanza del concepto de valencia de una forma completa y distinta a como lo presentan los textos, relacionándolo con el concepto de coordinación, con el fin probablemente, de explicar la formación de enlaces adicionales a los que puede formar un elemento si se sigue únicamente por los electrones de valencia o del último nivel de energía (concepto rígido y tradicional de valencia). El Docente \#2 aborda la enseñanza del concepto de valencia, relacionándolo con los electrones de las últimas capas de valencia, otra característica que el aborda hace referencia al número de enlaces que puede hacer un elemento, lo cual se dedujo de la expresión "Valencia es simplemente el número de uniones que puede hacer un átomo con otros átomos". El Docente \#3 aborda el concepto teniendo en cuenta la característica relacionada con la regla del octeto, como se expresó anteriormente. El Docente \#4 aborda las características de capacidad de combinación y estado de oxidación, lo cual se sustenta en lo que afirma el docente en su entrevista "para mí el concepto de valencia es la capacidad que tiene un átomo o un elemento, las posibilidades que tiene de combinarse con otros elementos de acuerdo a su carga positiva o su carga negativa". El Docente \#5, no enseña específicamente el concepto, sin embargo deja claro como característica del mismo, la capacidad de combinación de un átomo con la perdida, ganancia o compartición de electrones, sustentado en lo que dice en su entrevista "el concepto de valencia química está relacionado con la capacidad que tiene un átomo de formar enlaces con otro átomo bien sea por que le cedió electrones, le robó electrones o porque le dio electrones para compartirlo a través de en lace covalente".

Del análisis del mentefacto que se propone, se deducen las cualidades esenciales del concepto de valencia las cuales se encuentran al lado izquierdo del gráfico, (ver Figura 1), y las asumimos como las características del concepto de valencia que deben abordar los docentes para la enseñanza de este concepto. Los investigadores concebimos el concepto de valencia química como: La capacidad de enlace que tiene un átomo para formar una molécula (1), en el que el átomo puede ganar, perder o compartir uno o varios electrones de su último nivel de energía (2), los cuales ya hacían parte de ese nivel (número de oxidación) (3) o pudieron ser promovidos de niveles más internos y "sumarse" a los ya presentes (número de coordinación) (4).

Desde el punto de vista curricular se propone que los docentes involucren las características identificadas en el presente trabajo para la enseñanza del concepto de valencia, las cuales fueron organizadas en la herramienta propuesta, donde apoyados en el mentefacto, se deducen las cualidades esenciales del concepto de valencia, estas se encuentran al lado izquierdo del gráfico, las cuales se asumen como las características del concepto de valencia que deben abordar los docentes para la enseñanza de este concepto. Se concluye además que los mentefactos conceptuales, organizan las proposiciones y preservan los conocimientos allí almacenados, condensando una gran cantidad de información, al recurrir a diagramas visuales; lo que los constituye en poderosos condensadores cognitivos. Es decir siempre un tema, es sintetizable en pocas proposiciones, por lo que consideramos que si el estudiante domina los mentefactos esto le favorece el aprendizaje conceptual con mucho rigor.

\section{REFERENCIAS}

Carneiro, S.P. y R.A. Dai-Farra, As situações-problema no ensino de genética: estudando a mitose. Genética na Escola, 6(02), 30-34 (2011)

De Zubiría, M., Pedagogías del siglo XXI: Mentefactos I. El arte de pensar para enseñar y de enseñar para pensar, $2^{a}$ Edición, 240p. ECOE Ediciones, Bogotá, Colombia (2006)

Dos Santos-Fernandes, L. y A. Fernandes-Campos, Enseñanza del enlace químico desde una perspectiva situación-problema, Formación Universitaria, 7(6), 45-52 (2014)

Gallego, R. y otros cuatro autores, El concepto de valencia: su construcción histórica y epistemológica y la importancia de su inclusión en la enseñanza, Ciencia y educación, 10(3), 571-583 (2004)

Galperin, P.Y., Introducción a la psicología: un enfoque dialéctico, 1를 Edición, 160p. Ediciones Pablo del Rio S.A., Moscú, Rusia (1976)

Green, J.C., M.L.H. Green y G. Parkin, The occurrence and representation of three-centre two-electron bonds in covalent inorganic compounds, Chemical Communications, 48(94), 11481-11503 (2012)

Green, M.L.H. y G. Parkin, Application of the covalent bond classification method for the teaching of inorganic chemistry, Journal of Chemical Education, 91(6), 807-816 (2014) 
Ibañez, M., Mentefactos conceptuales como estrategia didáctico pedagógica de los conceptos básicos de la teoría de muestreo aplicados en investigación en salud, Rev. Cienc. Salud, 4(2), 62-72 (2006)

Latasa, I., P.Y. Lozano y N. Ocerinjauregi, Aprendizaje basado en problemas en currículos tradicionales: beneficios e inconvenientes, Formación Universitaria, 5(5), 15-26 (2012)

López, E. y J. Ospina, El concepto valencia: un abordaje histórico y la importancia de la inclusión histórica en la escuela secundaria, Memorias de la Maestría en Docencia de la Química - UNAL, 1(1), 75p. (2011)

Lorenzo, R.A., P. Fernández y A.M. Carro, Experiência en la aplicación del aprendizaje basado en problemas en la asignatura Proyecto de Licenciatura en Química, Formación Universitaria, 4(2), 37-44 (2011)

Lucca, N., Berríos, R. Investigación cualitativa: Fundamentos, diseños y estrategias, 2ª Edición. PR: Ediciones SM, Cataño, Puerto Rico (2009)

Lucca, N. y R. Berrios, Investigación Cualitativa en Educación y Ciencias Sociales, Publicaciones Puertorriqueñas, Puerto Rico (2003)

Simões, J.E., A.F. Campos y C.A. Marceiino-Jr, El uso de situaciones-Problema para la enseñanza superior de isomeria en la química inorgánica, Avances en Ciencias e Ingeniería, 4(2), $61-68$ (2013)

Tejada, C., C. Chicangana y A. Villabona, Enseñanza de la química basada en la formación por etapas de acciones mentales (caso enseñanza del concepto de valencia), Revista Virtual Universidad Católica del Norte, 38, 143-157 (2013)

Tobón, S., Formación basada en competencias, pensamiento complejo, diseño curricular y didáctica, $2^{\text {a }}$ Edición, 266p. ECOE Ediciones, Bogotá, Colombia (2005)

Vega, F. y otros tres autores, Experiencias de aprendizaje en Ingeniería Química; diseño, montaje y puesta en marcha de una unidad de destilación a escala de laboratorio mediante al aprendizaje basado en problemas, Formación Universitaria, 7(01) 13-22 (2014) 